\title{
Interleukin-1 receptor antagonist (IL1RN) is associated with suppression of early carcinogenic events in human oral malignancies
}

\author{
MASASHI SHIIBA ${ }^{1,2}$, KENGO SAITO ${ }^{1,3}$, HITOMI YAMAGAMI ${ }^{1}$, DAI NAKASHIMA ${ }^{1}$, \\ MORIHIRO HIGO $^{1}$, ATSUSHI KASAMATSU ${ }^{1}$, YOSUKE SAKAMOTO ${ }^{1}$, KATSUNORI OGAWARA ${ }^{1}$, \\ KATSUHIRO UZAWA $^{1}$, YUICHI TAKIGUCHI ${ }^{2}$ and HIDEKI TANZAWA ${ }^{1}$ \\ Departments of ${ }^{1}$ Oral Science, ${ }^{2}$ Medical Oncology, ${ }^{3}$ Molecular Virology, \\ Graduate School of Medicine, Chiba University, Chuo-ku, Chiba 260-8670, Japan
}

Received December 19, 2014; Accepted February 3, 2015

DOI: 10.3892/ijo.2015.2917

\begin{abstract}
Inflammatory abnormalities have been implicated in the pathogenesis of various human diseases, including cancer. Interleukin-1 receptor antagonist (IL1RN) is a potent anti-inflammatory molecule that modulates the biological activity of the proinflammatory cytokine, interleukin-1. The aim of this study was to examine the expression of IL1RN in oral squamous cell carcinomas (OSCCs), and to determine its clinical significance. Expression levels of ILIRN in matched normal and tumor specimens from 39 OSCCs were evaluated using real-time quantitative polymerase chain reaction methods, and immunohistochemical analysis. Protein expression of IL1RN was also examined in 18 oral premalignant lesions (OPLs). Expression of IL1RN mRNA was significantly downregulated in OSCCs compared with normal tissues. Decreased expression of IL1RN protein was also observed in OPLs and OSCCs. The IL1RN expression level was lower in the OPL cases with severe dysplasia compared to those with mild/moderate dysplasia. Significantly downregulated IL1RN expression was observed in all OSCC lesion sites examined when compared with the matched normal tissues. However, the decreased level of IL1RN expression did not correspond with tumor progression. Noteworthy, IL1RN expression was higher in the advanced OSCC cases (T3/T4) compared to early cases (T1/T2). Among OSCC samples, relatively higher IL1RN expression was associated with active tumor development in the OSCCs occurring in the buccal mucosa, oral floor, fauces
\end{abstract}

Correspondence to: Dr Masashi Shiiba, Department of Oral Science, Graduate School of Medicine, Chiba University, 1-8-1 Inohana, Chuo-ku, Chiba 260-8670, Japan

E-mail: m.shiiba@faculty.chiba-u.jp

Abbreviations: IL1RN, interleukin-1 receptor antagonist; OSCC, oral squamous cell carcinoma; OPL, oral premalignant lesion

Key words: interleukin-1 receptor antagonist, oral squamous cell carcinoma, oral premalignant lesion and gingiva, but not the tongue. These data suggest that IL1RN may exhibit opposing characteristics in oral malignancies depending on the stage of cancer development, suppressing early carcinogenic events, yet promoting tumor development in some lesion sites. Thus, IL1RN could represent a reliable biomarker for the early diagnosis of OSCCs. Furthermore, IL1RN may possess unknown and complex functions in the developed OSCC.

\section{Introduction}

Despite therapeutic and diagnostic advances, oral squamous cell carcinoma (OSCC) patients are often diagnosed at advanced stages, and therefore mortality rates remain high (1). OSCC carcinogenesis appears to evolve through a multistep process involving biomolecular changes, which results in the development of premalignant lesions and, subsequently, an invasive cancer. The identification of the molecular alterations associated with these events could yield insight into the mechanisms underlying the initiation and progression of this neoplasia, and provide new tools for the diagnosis, treatment, and prevention of OSCCs. To address this issue, we recently developed a strategy, using proteomics technologies to search for significant molecular biomarkers for oral carcinogenesis (2). Among the proteins identified was the interleukin-1 receptor antagonist (IL1RN), whose expression was found to be significantly downregulated in OSCC-derived cell lines compared to normal human oral keratinocytes.

It is generally accepted that inflammation is a strong risk factor for tumor development $(3,4)$. In oral cancer, chronic inflammation induced by prolonged exposure to alcohol, tobacco and pathogenic agents, has been considered to represent a potential common denominator in the development of tumors $(5,6)$. Interleukin-1 (IL-1) is a major proinflammatory cytokine, responsible for various acute and chronic inflammatory conditions. Evidence suggests that IL-1 may contribute to the promotion of tumor growth, angiogenesis, and metastasis in various human malignancies (7-9). IL1RN was initially characterized as a naturally occurring antagonist for IL-1. It shares $70 \%$ sequence homology with IL-1, and has the ability to bind 
to the membrane-anchored IL-1 receptor without initiating the IL-1 intracellular signaling cascade (10-12). Maintenance of an equilibrium between the IL-1 and IL1RN levels in local tissues influences the relative inflammatory effects of IL-1, and as reviewed by Arend in 2002, alteration of the balance predisposes cells to the development of a variety of diseases, including cancer (13).

Alterations in IL1RN expression could have an important role in oral carcinogenesis, however, little is known about the significance of IL1RN in OSCC. Therefore, the aim of the current study was to investigate the expression levels of IL1RN in a series of human primary OSCCs and oral premalignant lesions (OPLs), in order to establish a link between IL1RN expression and oral malignancies.

\section{Materials and methods}

Tissue specimens. Thirty-nine pairs of primary OSCC samples and corresponding normal oral epithelium tissue samples were obtained at the time of surgery performed at Chiba University Hospital between 2005 and 2009. The clinicopathological characteristics of the OSCC cases in this series are summarized in Table I. In addition, the 18 cases of advanced OPLs pathologically diagnosed as leukoplakia with epithelial dysplasia, i.e., mild $(n=1)$, moderate $(n=10)$, and severe $(n=7)$, in a high-risk oral site such as the ventrallateral tongue or gingiva, were obtained in the same manner. The resected tissues were divided into two parts: one part was frozen immediately after removal of the surrounding normal tissue, and was stored at $-80^{\circ} \mathrm{C}$ until extraction of its RNA was performed, and the second part was fixed in $10 \%$ buffered formaldehyde solution in preparation for pathological diagnosis and immunohistochemical (IHC) staining. The histopathological diagnosis of each tumor specimen was performed according to the International Histological Classification of Tumors, by the Department of Pathology, Chiba University Hospital. Clinicopathologic staging was determined by the TNM classification system of the International Union against Cancer.

Ethics statement. All patients provided written informed consent, in accordance with the protocol that was reviewed and approved by the Ethical Committee of Graduate School of Medicine, Chiba University (approval number, 236). The study was performed in accordance with the ethical standards of the Declaration of Helsinki.

Preparation of RNA. Total RNA was extracted using the TRIzol reagent (Invitrogen Life Technologies, Carlsbad, CA, USA), according to the manufacturer's instructions. Each extracted RNA or protein sample was stored separately at $-80^{\circ} \mathrm{C}$ until use.

mRNA expression analysis. Following RNA extraction and conversion of the extracted RNA to cDNA by reverse transcription, the mRNA expression levels of ILIRN were examined by real-time quantitative polymerase chain reaction methods (qRT-PCR) in OSCC specimens, and were compared with corresponding normal tissues. Before the cDNA synthesis, residual genomic DNA was removed from the total RNA by
Table I. Profiles of OSCC cases.

\begin{tabular}{|c|c|}
\hline Factors & Data \\
\hline \multicolumn{2}{|l|}{ Gender } \\
\hline Male & 26 \\
\hline Female & 13 \\
\hline \multicolumn{2}{|l|}{ Age } \\
\hline Min & 27 years \\
\hline Max & 87 years \\
\hline Average & 64.4 years \\
\hline SD & 13.1 \\
\hline \multicolumn{2}{|l|}{ Lesion sites } \\
\hline Tongue & 20 \\
\hline Gingiva & 8 \\
\hline Buccal mucosa & 4 \\
\hline Oral floor & 3 \\
\hline Fauces & 2 \\
\hline Lip & 1 \\
\hline Mandibular bone & 1 \\
\hline \multicolumn{2}{|l|}{ Differentiation } \\
\hline Well & 23 \\
\hline Moderately & 15 \\
\hline Poorly & 1 \\
\hline \multicolumn{2}{|l|}{ Primary tumor size } \\
\hline $\mathrm{T} 1$ & 1 \\
\hline $\mathrm{T} 2$ & 11 \\
\hline $\mathrm{T} 3$ & 17 \\
\hline $\mathrm{T} 4$ & 10 \\
\hline \multicolumn{2}{|c|}{ Regional lymph node metastasis } \\
\hline N0 & 18 \\
\hline N1 & 8 \\
\hline $\mathrm{N} 2$ & 15 \\
\hline \multicolumn{2}{|l|}{ Distant metastasis } \\
\hline No & 27 \\
\hline Unknown & 12 \\
\hline \multicolumn{2}{|l|}{ Stage } \\
\hline I & 1 \\
\hline II & 7 \\
\hline III & 10 \\
\hline IV & 21 \\
\hline \multicolumn{2}{|l|}{$\mathrm{pN}^{\mathrm{a}}$} \\
\hline No & 22 \\
\hline Yes & 17 \\
\hline
\end{tabular}

${ }^{\mathrm{a}} \mathrm{pN}$, Pathologically diagnosed regional lymph node metastasis.

DNase I treatment (DNA-free kit; Ambion, Austin, TX, USA). The nucleotide sequences of the gene-specific primers for qRT-PCR amplification of ILIRN were: forward 5'-TCCAAG CTCCATCTCCACTC-3', reverse 5'-GCTGAGTACCTGC 


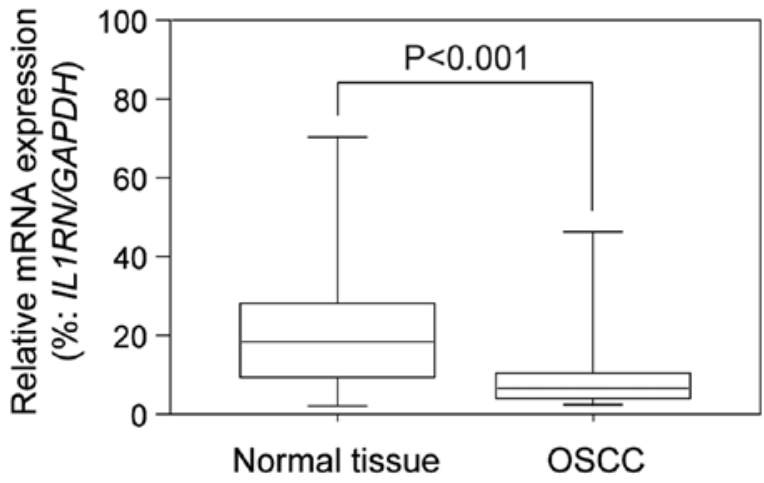

Figure 1. ILIRN mRNA expression in OSCC samples and corresponding normal tissues. qRT-PCR performed using RNA from OSCC samples and corresponding normal tissues, was applied to examine differences in the expression levels of ILIRN mRNA between the two tissues. The results are expressed as the means $\pm \mathrm{SD}$, and analyzed using unpaired Student's t-test.

CAAGAGC-3'. The glyceraldehyde-3-phosphate dehydrogenase gene (GAPDH; forward 5'-TTGGTATCGTGGA AGGACTGA-3', reverse 5'-TGTCATGATATTTGGCAG GTTT-3') was used as an internal control. The sequences of specific primers were checked before use by the Primer3 program (available at http:www-genome.wi.mit.edu/cgi-bin/ primer/primer3_www.cgi), to avoid amplification of genomic DNA or pseudogenes. Amplified products were analyzed by $3 \%$ agarose gel electrophoresis to ascertain their size and purity.

Real-time qRT-PCR was performed using the LightCycler FastStart DNA Master SYBR Green I kit (Roche, Indianapolis, IN, USA). To prepare the standard curve, $3 \mu \mathrm{g}$ of total RNA from normal oral tissue was reverse-transcribed with Superscript RT (Life Technologies) and oligo-d(T)12-18 primer, after which, serial dilutions were made corresponding to cDNA transcribed from $300,30,3.0$ and $0.3 \mathrm{ng}$ of total RNA. PCRs using the LightCycler apparatus were carried out in a final volume of $20 \mu \mathrm{l}$ of reaction mixture, consisting of $2 \mu \mathrm{l}$ of FirstStart DNA Master SYBR Green I mix, $3 \mathrm{mM} \mathrm{MgCl}{ }_{2}$ and $0.2 \mu 1$ of primers, according to the manufacturer's instructions. The reaction mixture was then loaded into glass capillary tubes. For real-time qRT-PCR, the DNA was subjected to an initial denaturation at $95^{\circ} \mathrm{C}$ for $10 \mathrm{~min}$, followed by 45 rounds of amplification at $95^{\circ} \mathrm{C}(10 \mathrm{sec})$ for denaturation, $58^{\circ} \mathrm{C}(10 \mathrm{sec})$ for annealing and $72^{\circ} \mathrm{C}$ for extension, with a temperature slope of $20^{\circ} \mathrm{C} / \mathrm{sec}$. The transcript levels of $I L 1 R N$ were estimated from the respective standard curves and normalized to the $G A P D H$ transcript in corresponding samples.

Immunohistochemistry (IHC). Immunohistochemical staining was performed using a series of OSCC specimens, which included 39 OSCCs with corresponding normal tissues, and 18 OPLs that were diagnosed histopathologically as leukoplakia with epithelial dysplasia. Considering that evidence has shown that the malignant transformation rate of oral leukoplakia with dysplasia is higher than that of oral leukoplakia without dysplasia (14), patients with advanced OPLs, defined as leukoplakia exhibiting epithelial dysplasia, were considered to be eligible for this study. IHC staining was carried out on $4 \mu \mathrm{m}$ sections of paraffin-embedded specimens. Briefly, after deparaffinization and hydration, the slides were pretreated in
$10 \mathrm{mM}$ sodium citrate buffer ( $\mathrm{pH}$ 6.0) in a microwave oven for $5 \min$ at $95^{\circ} \mathrm{C}$.

The endogenous peroxidase activity was quenched by a 30 -min incubation in a mixture of $0.3 \%$ hydrogen peroxide solution in $100 \%$ methanol. After washing with PBS buffer, the sections then were incubated with the primary antibody (biotinylated goat anti-human IL1RN antibody (1:100 dilution; R\&D Systems, Inc., Minneapolis, MN, USA) at room temperature and in a humidified chamber for $2 \mathrm{~h}$. After washing with PBS buffer, the slides were treated with peroxidase-labeled secondary antibody for $1 \mathrm{~h}$, followed by color development in 3,3'-diaminobenzidine tetrahydrochloride (Dako Japan Inc.). Finally, the slides were lightly counterstained with hematoxylin. As negative controls, slides were incubated with PBS instead of the primary antibody. To quantify the IL1RN protein expression, we used the Histo-score (H-score) system as previously described $(15,16)$. In brief, the mean percentage of epithelial cells exhibiting a persistent IL1RN signal was determined in at least five distinct fields using x400 magnification in each section. The intensity of the immunoreaction was scored as follows: 1+, weak; $2+$, moderate and $3+$, intense. Three target cell types: normal, premalignant and malignant epithelial cells, were identified for scoring.

In the present study, IL1RN immunoreactivity was detected mainly on the plasma membrane of target cells, and heterogeneously in the cytoplasm. The plasma membrane IL1RN immunoreaction was selected for the scoring. The percentage of IL1RN-positive cells and the staining intensity were then multiplied to produce the IL1RN H-score. The $\mathrm{H}$-scoring of each sample was performed by two independent pathologists, neither of whom had knowledge of the clinical status of the patients.

Statistical analysis. The statistical analyses of the expression levels of IL1RN mRNA and ILIRN protein were performed using unpaired or paired Student's t-test. $\mathrm{P}<0.05$ was considered to be statistically significant.

\section{Results}

ILIRN mRNA expression in OSCC tissues. According to real-time qRT-PCR data, the ILIRN mRNA expression in OSCC specimens was significantly $(\mathrm{P}<0.001)$ lower than that of the corresponding normal mucosa (Fig. 1). The relative ILIRN mRNA expression levels in normal and tumor sites ranged from 0.022 to 4.616 (median, 0.397 ) and 0.050 to 8.565 (median, 1.335), respectively.

ILIRN expression in OSCCs and OPLs. Representative results for IL1RN protein expression in normal oral tissues, OPLs and primary OSCCs are shown in Fig. 2. Normal oral mucosal specimens exhibited consistently strong IL1RN immunoreactivity on the plasma membrane of cells. According to the $\mathrm{H}$-scores, the expression levels of plasma membranous IL1RN were significantly reduced not only in OSCCs, but also in OPLs, in comparison to those of normal tissues (Fig. 3). In OPLs, although a case with mild dysplasia showed positive plasma membranous immunostaining for IL1RN, the staining signals decreased gradually in moderate through to severe dysplasia 
A
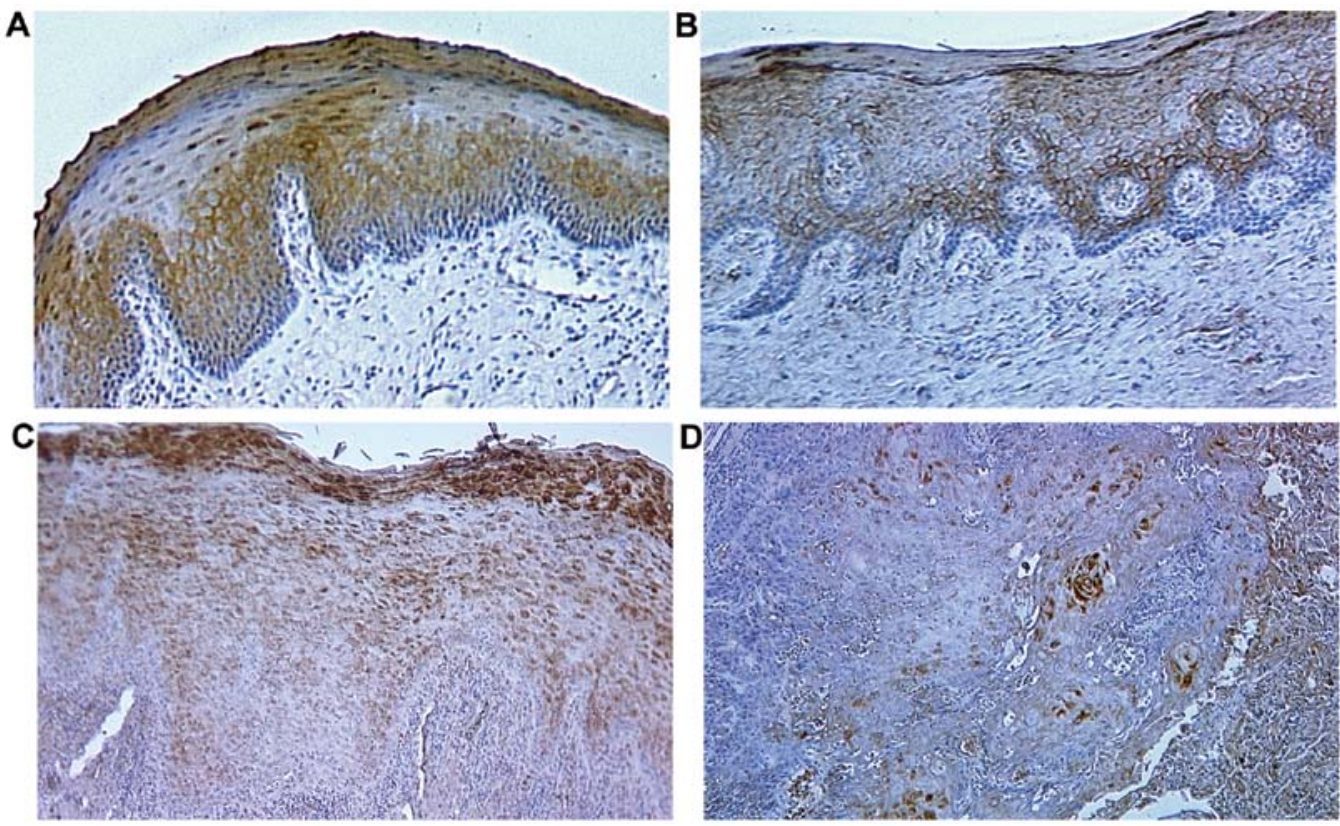

Figure 2. Representative results of immunohistochemical staining for IL1RN in normal tissues, OPLs and OSCCs. IHC staining of tissue sections shows IL1RN in brown. (A) Normal oral tissue. (B) OPL with mild dysplasia. (C) OPL with severe dysplasia. (D) OSCC. Original magnification was x200.

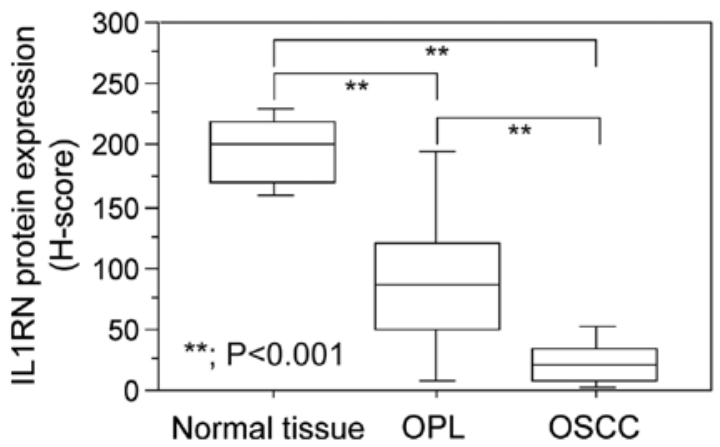

Figure 3. H-scores for the plasma membranous IL1RN expression in normal tissues, OPLs and OSCCs. To determine the extent of IL1RN expression across normal tissues, OPLs and OSCCs, H-scoring was performed on IL1RN stained tissues as described in Materials and methods. The results are expressed as the mean $\mathrm{H}$-score $\pm \mathrm{SD}$ for each tissue type, and analyzed using unpaired Student's t-test.

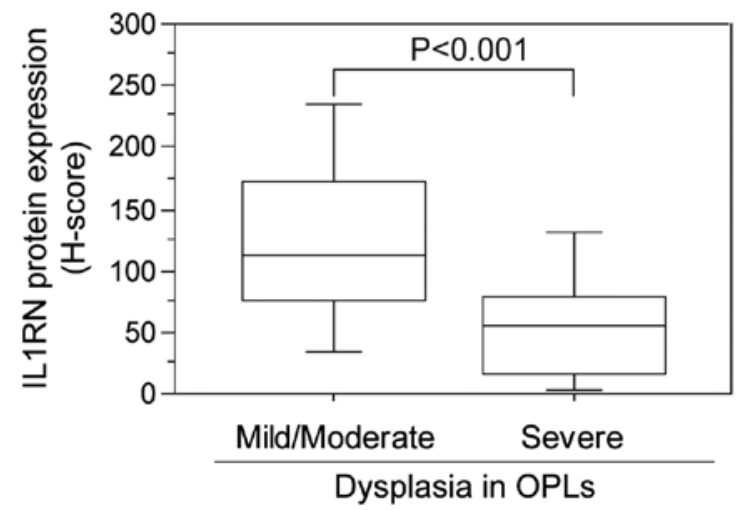

Figure 4. Correlation between IL1RN expression and the dysplasia grade in OPLs. Differences in IL1RN expression according to dysplasia grade in OPLs, i.e., mild/moderate dysplasia versus severe dysplasia, were determined by $\mathrm{H}$-scoring of IL1RN stained tissues. The results are expressed as the mean $\mathrm{H}$-score $\pm \mathrm{SD}$ for each tissue type, and analyzed using unpaired Student's t-test. cases. Significant differences were observed between mild/ moderate dysplasia cases and severe dysplasia cases (Fig. 4). Notably, IL1RN staining was also detected heterogeneously in the cytoplasm of OPL cells in some cases.

In OSCCs, the IL1RN immunoreactivity was largely lost in the specimens examined. Significantly downregulated IL1RN expression was observed in all lesion sites examined when compared with the matched normal tissues $(\mathrm{P}<0.001)$ (Fig. 5). However, the decreased level of IL1RN expression did not correspond with tumor progression (Fig. 6). Relative IL1RN expression levels, determined as relative values of $\mathrm{H}$-scores in tumor samples compared with normal tissues, were analyzed alongside clinicopathological factors. Noteworthy, IL1RN expression was higher in the advanced OSCC cases (T3/T4) in comparison to the early cases (T1/T2) ( $\mathrm{P}=0.0275)$ (Fig. 6A). Significant alteration of IL1RN associated with regional lymph node metastasis (Fig. 6B), TNM staging (Fig. 6C), differentiation (Fig. 6D) was not observed. OSCC cases were divided into two groups according to lesion sites: buccal mucosa/oral floor/fauces/gingival, and tongue. IL1RN expression associated with the clinicopathological factors was also analyzed separately, and the data revealed that higher IL1RN expression was associated with active tumor development: tumor size (Fig. 7A), regional lymph node metastasis (Fig. 7B), TNM staging (Fig. 7C), in the OSCC in buccal mucosa, oral floor, fauces and gingiva but not in the tongue. No significant difference was found between well and moderately/poorly differentiated OSCCs (Fig. 7D).

\section{Discussion}

A real-time qRT-PCR assay revealed that the relative $I L I R N$ mRNA expression levels were significantly lower in OSCCs than in their normal counterparts, which agrees with the recent study by Lallemant et al (17). Similarly, the IL1RN expression was significantly downregulated in the OSCC 

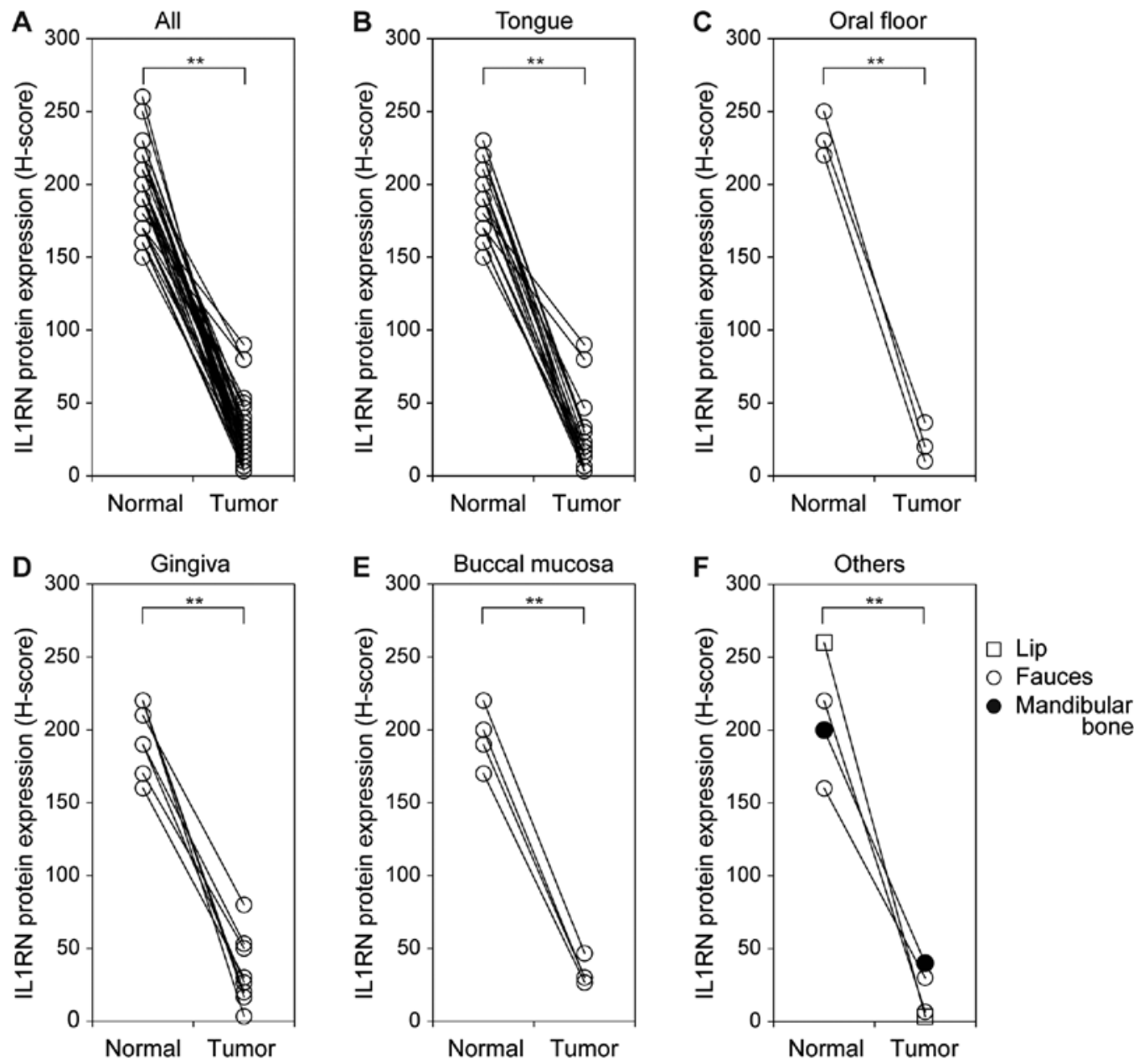

Figure 5. Comparison of IL1RN expression between OSCCs and the matched samples in each lesion site. IHC staining for IL1RN, and subsequent H-scoring, was performed on OSCCs $(\mathrm{T})$ and the normal matched samples $(\mathrm{N})$. H-scores for the matched $\mathrm{N}$ and $\mathrm{T}$ samples are plotted according to the lesion site from which they were acquired. Significant differences between the $\mathrm{N}$ and $\mathrm{T}$ of $\mathrm{H}$-scores for each lesion site, and across all samples (Total), are indicated $(\mathrm{P}<0.001$, paired Student's t-test)
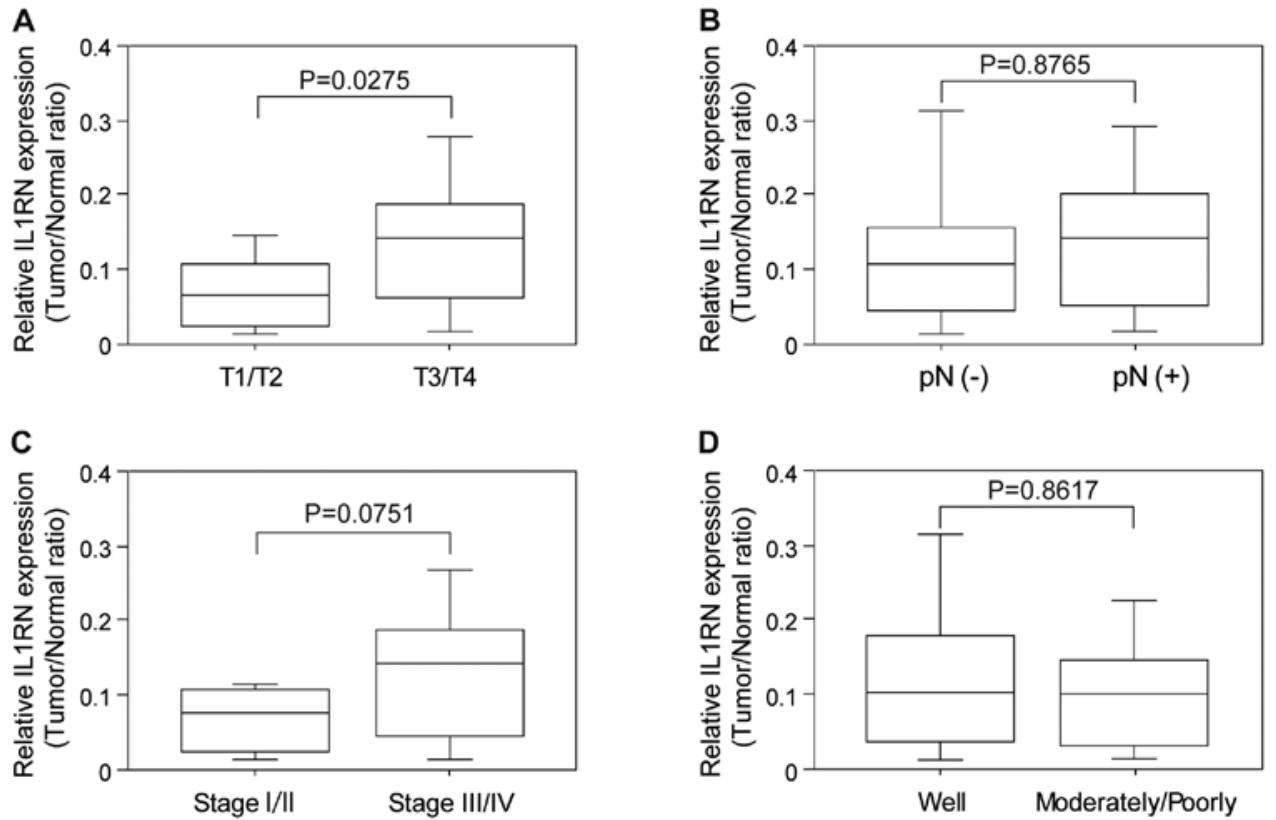

Figure 6. Analysis of IL1RN expression according to clinicopathological factors in OSCC cases. IL1RN expression levels in OSCCs determined as relative values of $\mathrm{H}$-scores in tumor samples compared with normal tissues, were further evaluated according to the following clinicopathological factors: primary tumor size (A), staging (B), pathologically determined metastasis of regional lymph nodes (C), differentiation (D). IL1RN expression was higher in the advanced OSCC cases (T3/T4) than the early cases (T1/T2). The results are expressed as the mean relative H-score \pm SD, and analyzed using unpaired Student's t-test. 

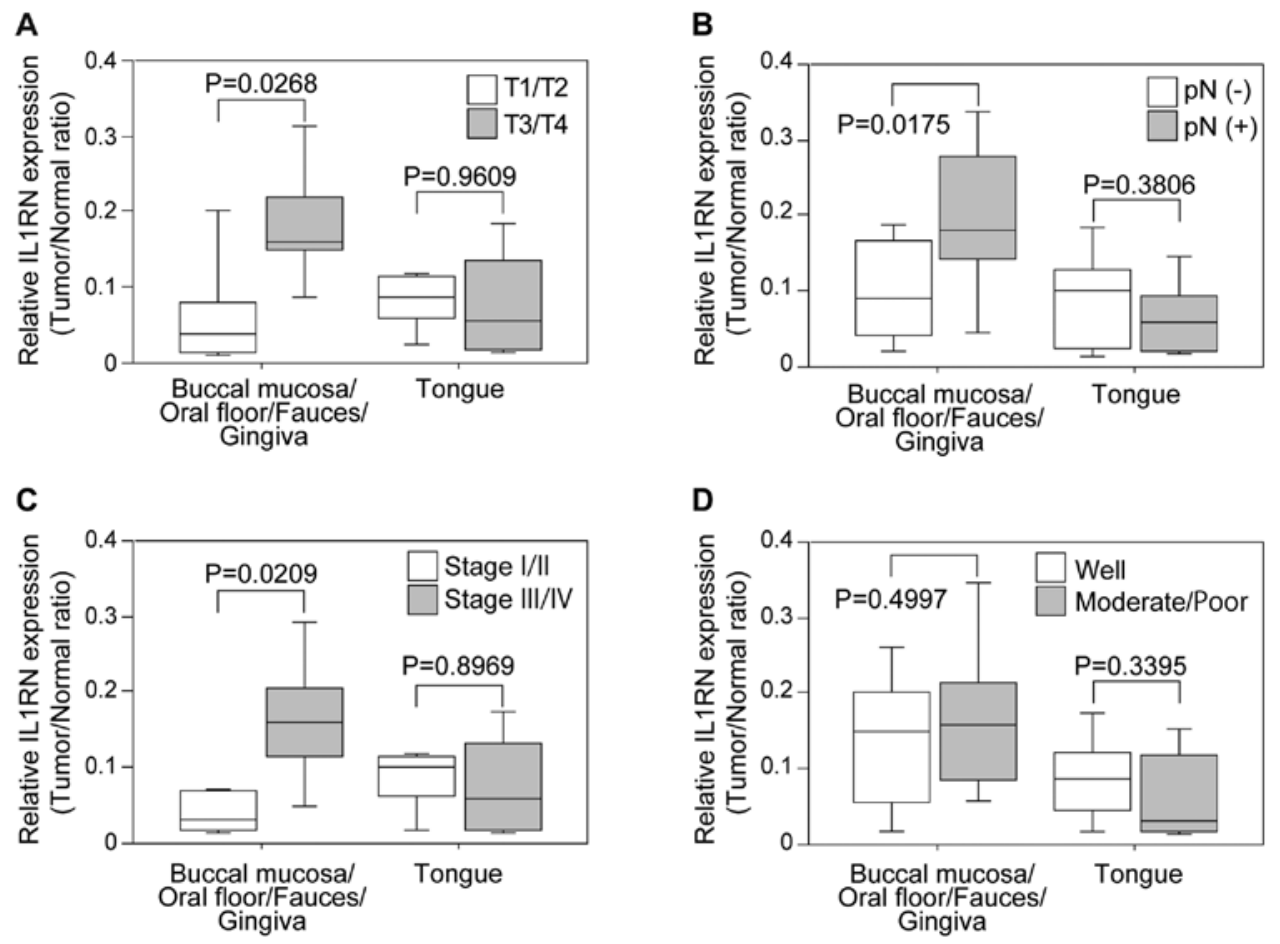

Figure 7. Analysis of lesion site-dependent IL1RN expression, associated with clinicopathological factors in OSCCs. Lesion site-dependent IL1RN expression levels determined as relative values of $\mathrm{H}$-scores in tumor samples compared with normal tissues, were further analyzed according to primary tumor size (A), staging (B), pathologically determined metastasis of regional lymph nodes (C), differentiation (D). In OSCC cases originated from buccal mucosa/oral floor/ fauces/gingiva advanced primary tumor size, staging and pathologically detected metastasis were closely associated with upregulated expression of IL1RN. However, no significant correlation between IL1RN expression and clinicopathological factors was found in tongue OSCC cases. The results are expressed as the mean relative $\mathrm{H}$-score $\pm \mathrm{SD}$, and analyzed using unpaired Student's t-test.

specimens examined compared to the normal oral epithelium. Yamamoto et al showed that in OSCCs, a significant loss of heterozygosity occurred at alleles in chromosome band $2 q 14$, where the IL1RN gene is located (18), and that genetic variations of IL1RN were strongly associated with the IL1RN expression level $(19,20)$. This suggested that altered IL1RN expression at the mRNA and protein levels could result from gene mutations in OSCC.

Low levels of IL1RN have been associated with greater disease severity in a variety of human malignancies, including leukemia (21), myeloma (22), colorectal cancer (23) and prostate cancer (24). In addition, experimental studies have shown that IL1RN decreases tumor growth or aggressive behavior by inhibiting IL-1-mediated activities in the cancer cells. Enhanced expression of IL1RN inhibits tumor growth of skin carcinoma cells in vitro and in vivo through blocking the transcriptional effect of IL-1 on cyclooxygenase-2, which is thought to play a pivotal role in tumor development (25). Elaraj et al showed that recombinant IL1RN significantly inhibited the tumor growth and metastatic potential of a human melanoma xenograft that constitutively secreted IL-1 (26). They also detected that IL1RN had anti-angiogenic effects in tumors, where it decreased the IL-1-mediated induction of angiogenic molecules, including interleukin-8 (IL-8) and vascular endothelial growth factor (VEGF). Similar findings were also observed for human breast cancer (27). These findings and our present results indicate that IL1RN may exhibit tumor-suppressing activity.

In contrast, there have been some contradictory observations that suggest IL1RN could increase tumorigenesis. For example, IL1RN was significantly overexpressed in cervical carcinomas compared with their normal counterparts (28). Additionally, IL1RN upregulation was also detected in gastric cancer, and was associated with a high incidence of metastases (29). Furthermore, in vitro studies showed that IL1RN enhances the growth and proliferation of human glioblastoma (30), prostatic (31) and hepatic cancer cells (32). Considering that IL-1 plays multiple biological roles in various tissues, the effect of IL1RN may be cell or tissue type-specific, or microenvironment-specific; for example, it may enhance cytotoxic T cell activity, and the tumoricidal capacities of natural killer (NK) cells $(33,34)$, and alterations in the properties of IL1RN are likely due to changes in the local IL-1-dependent pathways. Although the details of the biological mechanisms responsible for IL1RN alterations in tumors have not been determined, our results suggest that IL1RN may be critical for maintaining the normal condition of cells, and that a loss of the expression of the protein could result in an enhanced risk of cancer development in the oral cavity.

The data described in the present study also showed that the IL1RN expression was gradually downregulated in accordance with the degree of dysplasia. As oral dysplasia shows a significant rate of transformation to cancer $(35,36)$, there is an urgent need to identify better ways to predict which patients with dysplastic precursor lesions will develop OSCC. The present results suggest that IL1RN may suppress the early carcinogenic events and that the expression level could represent a useful biomarker for the early diagnosis of OSCC. However, our data revealed that relatively higher expression of 
IL1RN is found in the developed OSCC samples, especially in the OSCC occurring in buccal mucosa, oral floor, fauces and gingiva but not in the tongue.

In conclusion, IL1RN may exhibit conflicting characteristics in controlling oral malignancies; namely, suppressive activity in the early carcinogenic events, but also lesion site-dependent tumor development promotion. Thus, IL1RN should be a reliable biomarker for the early diagnosis of OSCC. However, IL1RN may have unknown and complicated functions in the developed OSCC, suggesting that further investigation is necessary before considering IL1RN as a therapeutic target in oral cancer.

\section{References}

1. La Vecchia C, Lucchini F, Negri E and Levi F: Trends in oral cancer mortality in Europe. Oral Oncol 40: 433-439, 2004.

2. Koike H, Uzawa K, Nakashima D, Shimada K, Kato Y, Higo M, Kouzu Y, Endo Y, Kasamatsu A and Tanzawa H: Identification of differentially expressed proteins in oral squamous cell carcinoma using a global proteomic approach. Int J Oncol 27: 59-67, 2005.

3. Sethi G, Shanmugam MK, Ramachandran L, Kumar AP and Tergaonkar V: Multifaceted link between cancer and inflammation. Biosci Rep 32: 1-15, 2012.

4. Aggarwal BB and Gehlot P: Inflammation and cancer: How friendly is the relationship for cancer patients? Curr Opin Pharmacol 9: 351-369, 2009.

5. Patel MM and Pandya AN: Relationship of oral cancer with age, sex, site distribution and habits. Indian J Pathol Microbiol 47: 195-197, 2004.

6. Znaor A, Brennan P, Gajalakshmi V, Mathew A, Shanta V, Varghese $C$ and Boffetta P: Independent and combined effects of tobacco smoking, chewing and alcohol drinking on the risk of oral, pharyngeal and esophageal cancers in Indian men. Int J Cancer 105: 681-686, 2003.

7. Matsuo Y, Sawai H, Ma J, Xu D, Ochi N, Yasuda A, Takahashi H, Funahashi H and Takeyama H: IL-1alpha secreted by colon cancer cells enhances angiogenesis: The relationship between IL-1alpha release and tumor cells' potential for liver metastasis. J Surg Oncol 99: 361-367, 2009.

8. Matsuo Y, Sawai H, Ochi N, Yasuda A, Takahashi H, Funahashi H, Takeyama H and Guha S: Interleukin-1alpha secreted by pancreatic cancer cells promotes angiogenesis and its therapeutic implications. J Surg Res 153: 274-281, 2009.

9. Ma J, Sawai H, Matsuo Y, et al: Interleukin-1alpha enhances angiogenesis and is associated with liver metastatic potential in human gastric cancer cell lines. J Surg Res 148: 197-204, 2008.

10. Dinarello CA: Interleukin-1 and its biologically related cytokines. Adv Immunol 44: 153-205, 1989.

11. Dinarello CA: Interleukin-1, interleukin-1 receptors and interleukin-1 receptor antagonist. Int Rev Immunol 16: 457-499, 1998.

12. Arend WP: Interleukin 1 receptor antagonist. A new member of the interleukin 1 family. J Clin Invest 88: 1445-1451, 1991.

13. Arend WP: The balance between IL-1 and IL-1Ra in disease. Cytokine Growth Factor Rev 13: 323-340, 2002.

14. Amagasa $T$, Yamashiro $M$ and Ishikawa $H$ : Yamashiro $M$ and and Ishikawa $\mathrm{H}$ : Oral leukoplakia related to malignant transformation. Oral Sci Int 3: 45-55, 2006.

15. Bilalovic N, Sandstad B, Golouh R, Nesland JM, Selak I and Torlakovic EE: CD10 protein expression in tumor and stromal cells of malignant melanoma is associated with tumor progression. Mod Pathol 17: 1251-1258, 2004.

16. McCarty KS Jr, Szabo E, Flowers JL, et al: Use of a monoclonal anti-estrogen receptor antibody in the immunohistochemical evaluation of human tumors. Cancer Res 46 (Suppl 8): S4244-S4248, 1986.

17. Lallemant B, Evrard A, Combescure C, et al: Clinical relevance of nine transcriptional molecular markers for the diagnosis of head and neck squamous cell carcinoma in tissue and saliva rinse. BMC Cancer 9: 370, 2009.
18. Yamamoto N, Mizoe J, Numasawa H, Tsujii H, Shibahara T and Noma H: Allelic loss on chromosomes 2q, 3p and 21q: Possibly a poor prognostic factor in oral squamous cell carcinoma. Oral Oncol 39: 796-805, 2003.

19. Dewberry R, Holden H, Crossman D and Francis S: Interleukin-1 receptor antagonist expression in human endothelial cells and atherosclerosis. Arterioscler Thromb Vasc Biol 20: 2394-2400, 2000.

20. Rafiq S, Stevens K, Hurst AJ, et al: Common genetic variation in the gene encoding interleukin-1-receptor antagonist (IL-1RA) is associated with altered circulating IL-1RA levels. Genes Immun 8: 344-351, 2007.

21. Kurzrock R: Cytokine deregulation in cancer. Biomed Pharmacother 55: 543-547, 2001.

22. Gherardi RK, Bélec L, Soubrier M, Malapert D, Zuber M, Viard JP, Intrator L, Degos JD and Authier FJ: Overproduction of proinflammatory cytokines imbalanced by their antagonists in POEMS syndrome. Blood 87: 1458-1465, 1996.

23. Iwagaki H, Hizuta A and Tanaka N: Interleukin-1 receptor antagonists and other markers in colorectal cancer patients. Scand J Gastroenterol 32: 577-581, 1997.

24. Parekh DJ, Ankerst DP, Baillargeon J, Higgins B, Platz EA, Troyer D, Hernandez J, Leach RJ, Lokshin A and Thompson IM: Assessment of 54 biomarkers for biopsy-detectable prostate cancer. Cancer Epidemiol Biomarkers Prev 16: 1966-1972, 2007.

25. La E, Rundhaug JE and Fischer SM: Role of intracellular interleukin-1 receptor antagonist in skin carcinogenesis. Mol Carcinog 30: 218-223, 2001.

26. Elaraj DM, Weinreich DM, Varghese S, Puhlmann M, Hewitt SM, Carroll NM, Feldman ED, Turner EM and Alexander HR: The role of interleukin 1 in growth and metastasis of human cancer xenografts. Clin Cancer Res 12: 1088-1096, 2006.

27. Lindahl G, Saarinen N, Abrahamsson A and Dabrosin C: Tamoxifen, flaxseed, and the lignan enterolactone increase stroma- and cancer cell-derived IL-1Ra and decrease tumor angiogenesis in estrogen-dependent breast cancer. Cancer Res 71: 51-60, 2011.

28. Fujiwaki R, Iida K, Nakayama K, Kanasaki H, Hata K, Katabuchi H, Okamura $\mathrm{H}$ and Miyazaki K: Clinical significance of interleukin-1 receptor antagonist in patients with cervical carcinoma. Gynecol Oncol 89: 77-83, 2003.

29. Iizuka N, Hazama S, Hirose K, Abe T, Tokuda N, Fukumoto T, Tangoku A and Oka M: Interleukin-1 receptor antagonist mRNA expression and the progression of gastric carcinoma. Cancer Lett 142: 179-184, 1999.

30. Oelmann E, Kraemer A, Serve H, Reufi B, Oberberg D, Patt S, Herbst H, Stein H, Thiel E and Berdel WE: Autocrine interleukin-1 receptor antagonist can support malignant growth of glioblastoma by blocking growth-inhibiting autocrine loop of interleukin-1. Int J Cancer 71: 1066-1076, 1997.

31. Hsieh TC and Chiao JW: Growth modulation of human prostatic cancer cells by interleukin-1 and interleukin-1 receptor antagonist. Cancer Lett 95: 119-123, 1995.

32. Yamada Y, Karasaki H, Matsushima K, Lee GH and Ogawa K: Expression of an IL-1 receptor antagonist during mouse hepatocarcinogenesis demonstrated by differential display analysis. Lab Invest 79: 1059-1067, 1999.

33. Dvorkin T, Song X, Argov S, White RM, Zoller M, Segal S, Dinarello CA, Voronov E and Apte RN: Immune phenomena involved in the in vivo regression of fibrosarcoma cells expressing cell-associated IL-1alpha. J Leukoc Biol 80: 96-106, 2006.

34. Voronov E, Weinstein Y, Benharroch D, et al: Antitumor and immunotherapeutic effects of activated invasive $\mathrm{T}$ lymphoma cells that display short-term interleukin 1alpha expression. Cancer Res 59: 1029-1035, 1999.

35. Al-Dakkak I: Oral dysplasia and risk of progression to cancer. Evid Based Dent 11: 91-92, 2010.

36. Liu W, Bao ZX, Shi LJ, Tang GY and Zhou ZT: Malignant transformation of oral epithelial dysplasia: Clinicopathological risk factors and outcome analysis in a retrospective cohort of 138 cases. Histopathology 59: 733-740, 2011. 